\title{
Prevention of the Stigma of Mental Disorders in the Community
}

\author{
Retno Puji Astuti, Antonia Rensiana Reong, Fakhrun Nisa' Fiddaroini and M. Elyas Arif \\ Budiman
}

Faculty of Nursing, Universitas Airlangga, Surabaya, Indonesia

\section{ABSTRACT}

Introduction: The stigma felt towards clients with mental disorders is stil negative in community. Health education programs are run by health workers in order to reduce the stigma for clients with mental disorders in the community. These programs have been widely implemented but there has been a low impact on the community thus far. The aim of this study was to gain an insight into the stigma focused on clients with mental disorders in the community.

Methods: The databases used were SCOPUS, ProQuest, SAGE and Science Direct in order to identify potential articles. The articles were limited to being published in a range of 5 years from 2013 to 2018. The keywords used in the literature review were "stigma" AND "community" AND "mental disorder".

Results: This study reports on the latest experience of stigma in relation to clients with mental disorders. The stigma is given from the community to the client with a mental disorder at 4 levels, namely labeling, stereotype, exclusion and discrimination. This makes the social life of the clients disturbed. Stigma and the prevention of it must therefore be further developed in the community.

Conclusion: From this systematic review should be considered to reduce and prevent stigma in clients with mental disorders.
\end{abstract}

\section{ARTICLE HISTORY}

Received: December 26, 2019

Accepted: December 31, 2019

\section{KEYWORDS}

Mental disorder; stigma; prevention;

\section{CONTACT}

Retno Puji Astuti

$\triangle$ retno.puji.astuti-

2018@fkp.unair.ac.id

$\doteq$ Faculty of Nursing, Universitas

Airlangga, Surabaya, Indonesia

Cite this as: Astuti, R. P., Reong, A. R., Fiddaroini, F. N., \& Budiman, M. E. A. (2019). Prevention of the Stigma of Mental Disorders in the Community. Jurnal Ners, 14(3si), 165-170.doi:http://dx.doi.org/10.20473/jn.v14i3(si).16958

\section{INTRODUCTION}

Stigma has been linked to adverse experiences for people with mental disorder as it acts as a barrier to help-seeking and it can affect all aspects of a person's life (Corker et al., 2015)(Subramaniam et al., 2017). Stigma is referred to as a social construct comprised of 4 interrelated components including: 1) people distinguish and label human differences as a knowledge problem; 2) labeled people are caused damage by negative stereotypes as a attitude problem; 3) labeled people are placed in exclusion and 4) labeled people have a lost status and are discriminated against in terms of behavior problems (Svensson \& Hansson, 2016)(Reavley, Morgan, \& Jorm, 2017). A stigmatized attitude was related to the mental disorder itself (Zhuang, Wong, Cheng, \& Pan, 2017). The stigma of mental health has a detrimental effect because the sufferers tend to be hesitant to reveal a psychiatric diagnosis, meaning that there is a decrease in mental health-seeking behavior and decreased medication adherence, which potentially worsens the disease prognosis (Ndetei et al., 2016)(Subica et al., 2019).

When studying the public stigma of people with mental disorder, several factors such as gender, age, socioeconomic status, knowledge of mental disorder and contact or familiarity with individuals with a mental disorder should be considered (Lien \& Kao, 2019). Some of studies state that culture influences experience, expression and the determinants of stigma and the effectiveness of the various approaches to reducing stigma. A meaningful intervention to combat stigma must, therefore, consider the cultural context (Ikwuka et al., 2016). An INDIGO schizophrenia study involved 732 individuals diagnosed with schizophrenia in 27 countries where $47 \%$ of participants experienced discrimination when making or maintaining friends, $43 \%$ experienced discrimination from their family members and $29 \%$ found keeping work to be difficult. The same group examined the discrimination experienced by 1087 people who were diagnosed with major depressive disorder in 35 countries and found that $79 \%$ of participants experienced 
discrimination in at least one area of life in Indonesia (Farrelly et al., 2014).

Recent research more often investigates the level of stigma and discrimination using direct reports from the users of mental health services where the results of these studies show a high level of stigma and discrimination, regardless of the type of diagnosis (Corker et al., 2015). Public stigma is still high despite efforts to improve public knowledge and attitudes and to reduce discrimination (Aznar-Lou, SerranoBlanco, Fernández, Luciano, \& Rubio-Valera, 2016). Several recent studies on reducing stigma have found that most programs aim to increase awareness of the mental disorder and to improve mental health literacy. Countries that have carried out anti-stigma campaign activities have proven there to be positive results in terms of reducing the negative attitudes towards people with a mental disorder (Corker et al., 2015).

One study showed that there is a positive effect from the anti-stigma campaign over a 2-year multimedia-based period where the standardized tools captured data on knowledge, attitude and the behavior towards mental health as well as the perceptions related to help-seeking for a mental disorder (Maulik et al., 2019). Attitudes toward someone with a mental disorder can be improved by providing information continuously related to said mental disorder (Schomerus et al., 2016). The aim of this systematic review should be considered to reduce and prevent stigma in clients with a mental disorder.

\section{MATERIALS AND METHODS}

\section{Data sources and search}

The journal search strategy begins with asking research question "what stigma of mental disorder in the community?" It was reviewed in actions to reduce stigma in clients with mental disorders in the community. The databases used were SCOPUS, ProQuest, SAGE and Science Direct. The articles were limited to a publishing range of 5 years from 2014 to 2019 within the areas of nursing, psychology and medicine in English language journals. The keyword used in the literature review were "stigma" AND "community" AND "mental disorder".

\section{Article selection criteria}

The feasibility of this study was assessed using the PICOT approach. The population was clients with mental disorders. The intervention used was an antistigma health education campaign. There was no comparison. The expected output was a positive effect in terms $\mathrm{f}$ an increase in people's knowledge, attitudes and behavior in terms of stigmatizing clients with mental disorders.

The inclusion criteria were 1) all articles related to the act of reducing the stigma of clients with mental disorders in the community; 2) the population in the article was the clients with mental disorders and the community; 3 ) the actions taken in the articles are health education interventions that can reduce stigma and 4) the results measured in the article show an increase in the positive effects of anti-stigma campaigns.

\section{Exclusion data}

The exclusion criteria for this study were non-health education interventions and journals published before 2014 .

\section{Research design}

Most of the 15 articles in the review used a crosssectional design.

\section{Data Items}

The following information will be collected from each paper: (1) author(s); (2) year of publication; (3) journal title; (4) summary or journal paper; (5) design and setting; (6) country; (7) study objective(s); (8) theory and/or hypothesis; (9) definitions of stigma; (10) instruments for data collection; (11) samples of demographic information (age, gender, etc.) and (12) the type of participants, sample size and the time of the data collection.

\section{Risk of Bias in Individual Studies}

The credibility of the synthesis can be compromised by reporting on the bias that arises when the results of the study are influenced by the nature of the results of the study. For example, there may be bias because of selective publication, such as where a study is only published if the findings are considered to be interesting(Page, McKenzie, \& Higgins, 2018). Risk of bias was considered during the search for research and during the quantitative synthesis of the research selected(Larkings \& Brown, 2018).

No formal assessment of risk of bias was undertaken due to the limited articles available that explored the relationship between an anti-stigma campaign and stigma among people with mental illness in community. Instead, all of the articles that met the inclusion criteria were included, and several factors that influence the risk of bias were discussed throughout the Results and Discussion sections in the present study, such as the characteristics of the included studies, the steps used for reducing stigma and the multiple terms and definitions used for stigma, people with mental illness, and the antistigma campaign itself (Larkings \& Brown, 2018).

\section{Data Synthesis}

In the final report, we will present a domain of the definitions in the form of a list with various subgroups. The papers will be divided into these subgroups on the basis of healthy people, at risk people and people with mental disorders where the participants came from both men and women, and teenagers and adults. We will examine the anti-stigma campaigns used to reduce stigma in the community. We will record the various stigmas in our table of results. The various anti-stigma campaigns will then be compared and contrasted. The collected data will also be used to demarcate the various chronological trends within the anti-stigma campaigns. Subsequently, the data will be combined and 
categorized based on the procedure mentioned above.

\section{RESULTS}

\section{Results of Search}

The researcher identified 2.354 citations, with 354 being through the initial stages of feasibility assessment using study type, title and abstract. The first step that the researchers did was to examine the various articles manually that were related to the research title. Of the 354 potentially relevant articles, only 28 were selected for further assessment. Detailed reviews of the remaining 24 articles were carried out and 4 further articles were excluded because they had the same title. Overall, articles were excluded for the following reasons: they did not involve an anti-stigma health campaign for the clients with mental disorders and there was no positive effect from the intervention. Of the 24 remaining papers, a total of 15 studies were examined in detail that met the expected criteria.

Table 1. Literature Search Summary

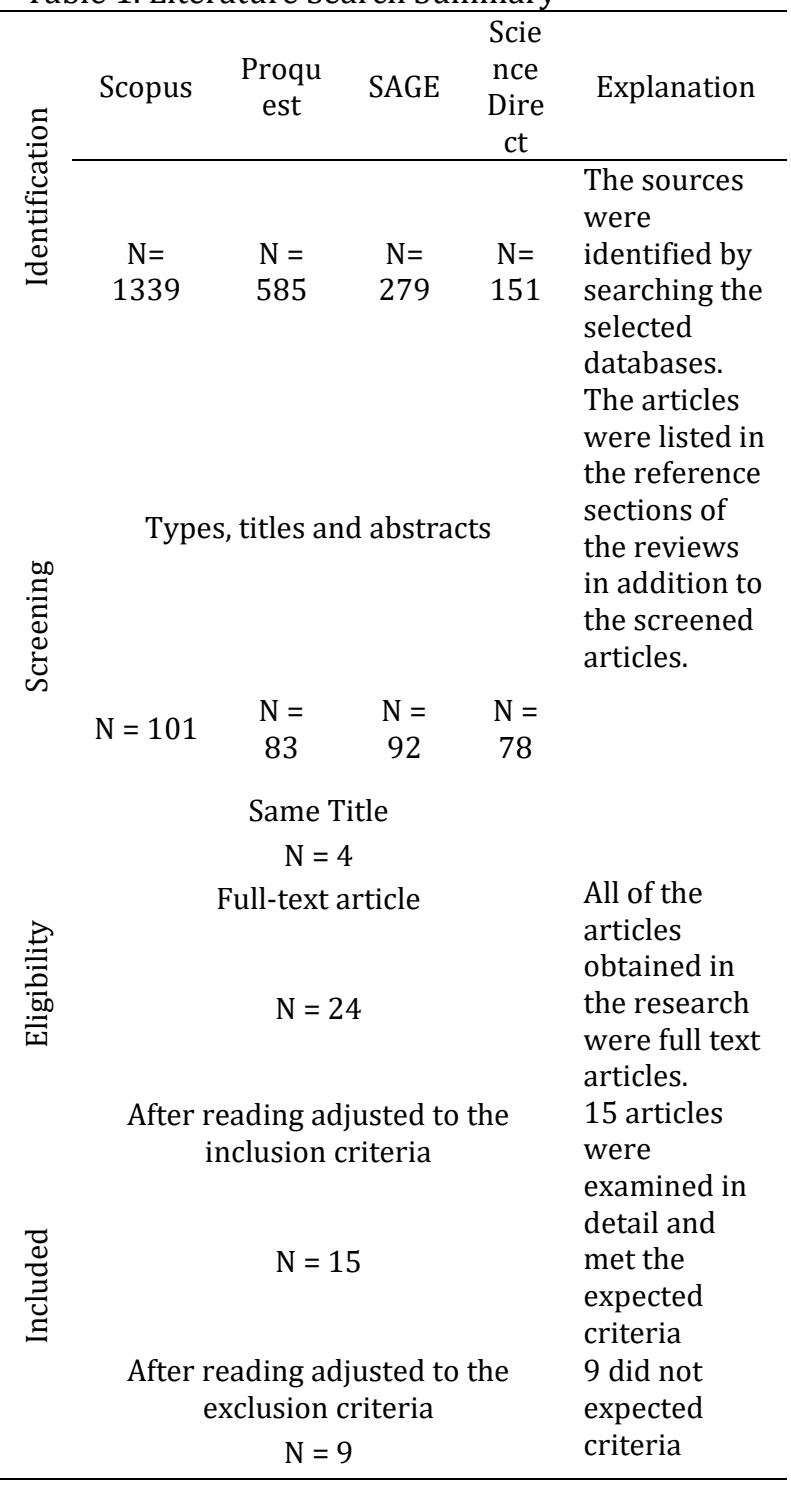

\section{Sample characteristics}

The samples used came from various countries where the participants were taken from those aged 18 and above. The participants from the various communities were healthy people, at risk people and people with mental disorders. The participants were both men and women and both teenagers and adults. All of the participants provided written informed consent including parental written consent where appropriate (Xu et al., 2016)(Townley, Brusilovskiy, \& Salzer, 2017). Articles were included in this review if they focused on evaluating the stigma toward adults and/or children with a diagnosis of mental disorder included quantitative or qualitative measures of public stigma. They also had to be written in English.

\section{Types of interventions}

The results of the review of the research articles that met the inclusion criteria showed that there are various types of intervention to know or prevent stigma in the community, such as questionnaires, interviews and Mental Health First Aid (MHFA). The settings from the 15 research articles were in the community. From the review of the 15 research articles, there was 1 research article that was quasiexperimental and 14 research articles that were cross-sectional.

\section{Procedures}

Most of the research was carried out in Europe and Asia. The quantitative studies used questionnaires developed by the authors or adaptations of instruments previously designed. The qualitative studies collected information from interviews, both face to face and over the telephone. The standardized tool captures looked at the knowledge, attitude and behavior towards mental health as well as the perceptions related to help-seeking for mental disorder patients.

\section{Relation with a mental disorder}

In this study, the community was divided into 2 types, namely people who have direct contact with an MD (family, friends, health worker) and people who are not directly related to an MD (knowing of MDs through lessons or the news). One study showed that relating to someone with $\mathrm{MD}$ is associated with a better attitude and behavior (Aznar-Lou et al., 2016). The criterion for the participants was also based on education being divided into two, namely the participants with low education (zero to secondary education) and the participants with higher education (postsecondary education). Marriage status was also identified as married and unmarried respectively.

\section{Type of questionnaire}

The questionnaires used in the study were Community Attitudes to Mental Illness (CAMI), the Reported and Intended Behavior Scale (RIBS), the Discrimination and Stigma Scale (DISC-12), Mental Illness-Related Investigations on Discrimination (MIRIAD), the Depression Literacy Questionnaire, the 
Perceived Devaluation Discrimination Questionnaire, the Stigma Stress Scale and the Positive and Negative Syndrome Scale.

\section{DISCUSSION}

Attitudes towards people with mental disorders are varied(Aznar-Lou et al., 2016). People tend to hesitate to express their mental disorder to their family and friends because they are worried whether their family and friends will understand and accept the situation. This has an effect on the increasing duration of the disease which will increase the experience of discrimination(Corker et al., 2015). For example, people with a diagnosis of their first depressive episode reported experiencing discrimination in areas of life that were far more significant than the participants diagnosed with schizophrenia(Svensson \& Hansson, 2016). Higher levels of discrimination experienced by people with a disorder other than depression or anxiety are consistent. This shows that people are more likely to have a stigmatizing attitude towards people with schizophrenia because of the duration of the disease(Farrelly et al., 2014).

The general public regards depression as a less serious condition. It is seen of as an old disease that is not worth getting care and assistance for as much as other types of mental disorder. The behavior of people diagnosed with depression may be less supportive and more discriminatory(Farrelly et al., 2014). This can be considered negative and discriminatory by the participants with a diagnosis of depression. The higher discrimination of participants with depression can also be explained by the duration and/or severity of the disease. The public stigma present means that other people will view it negatively if they seek treatment and this is associated with a reluctance to seek professional help(Lien \& Kao, 2019).

Anti-stigmatic interventions should aim to directly influence emotions and to increase the social acceptance of those affected. Emotional reactions that are stronger predict discrimination rather than stereotypes. There are several ways to fight authoritarianism such as contact-based education or education to increase knowledge, attitudes, mental health literacy and to reduce stereotypes, in addition to the desire for interpersonal distance(Aznar-Lou et al., 2016). Social contact is considered to be the most effective intervention used as a part of anti-stigma campaigns(Maulik et al., 2019).

Younger people are more tolerant and less stigmatized because of the changing knowledge and perceptions of mental disorder. Young people at risk of psychosis tend to seek professional help because they may not want to be labeled as a mental disorder patient and suffer prejudice and discrimination (Subramaniam et al., 2017). This could also be due to the fact that younger people know more about the causes, treatment and results of mental disorder as a result of campaign exposure in their place of education, as well as through social media. Those who have personal contact with someone who has a mental disorder have a better understanding and they are more sensitive to stigma and discrimination against people with mental disorders(Svensson \& Hansson, 2016). Those with a higher education level have more knowledge and a better understanding of people with mental disorders(Farrelly et al., 2014). If they have family members who suffer from a mental disorder, then they will likely experience stigmatization which leads to empathy and nonstigma. Social contact with people with mental disorder is an effective anti-stigma intervention as well.

Working with mentally disturbed individuals is very important to encourage them to identify and access the community resources that facilitate activity participation and community involvement. One method is utilizing peer support and strengthening social support networks. This helps individuals to identify opportunities to participate in their environment.

This systematic review provides evidence about the effectiveness of anti-stigma campaign interventions as one of health education steps in preventing and reducing the stigma felt by the clients with mental disorders. The results of the review explain that one effective way that can be used to carry out the anti-stigma campaign is used multimedia. The results of this review are useful for both clinical nurses and community mental nurses.

The results of this review can be implicated in providing anti-stigma campaigns where the campaign is effective at preventing the stigma that develops in the community with clients with mental disorders. Health workers can provide structured education to ordinary people about the importance of supporting mental health improvement, so the clients can socialize within the community and get maximum care.

Nurses can implement health interventions to reduce stigma by using technology, especially social media. Better conditions in the application of health education can prevent the emergence of stigma and improve the quality of social life of clients with mental disorders, for example, in terms of work and family. Several studies have explained that anti-stigma campaigns should be carried out with regard to the local culture. A positive attitude towards someone with a mental disorder can be improved by providing information from the anti-stigma campaigns continuously in a manner that is related to mental disorders.

A limitation of this research is that the data is not representative of any of the participating countries, so it can thus carry a higher risk of bias. This is because the culture, social life and the level of stigma in each region is different. Further research is essential to explore the utilized programs in order to increase the self-awareness of the risk of mental disorders without stigmatizing connotations as well as strategies to reduce the stigma for people at risk of mental disorders. 


\section{CONCLUSION}

The results of the systematic reviewing of 15 research journals related to the stigma of clients with mental disorders in the community were that the implementation of nursing services can be used to reduce stigma in the form of an anti-stigma campaign. This has been proven to reduce discrimination relating to the neighbor's life, dating, education, marriage, religious activities and the health of the client with a mental disorder.

Future actions aimed at reducing stigma in mental health must be directed at reducing the component of authoritarianism from the stigma itself. Public campaigns can be an important way to combat stigma in mental health and the campaigns can have a greater impact by targeting older people, people who have a lower educational attainment and people who have no contact with someone with a mental disorder.

\section{REFERENCES}

Aznar-Lou, I., Serrano-Blanco, A., Fernández, A., Luciano, J. V., \& Rubio-Valera, M. (2016). Attitudes and intended behaviour to mental disorders and associated factors in catalan population, Spain: Cross-sectional population-based survey. $B M C$ Public Health, 16(1), 1-12. https://doi.org/10.1186/s12889-016-2815-5

Corker, E. A., Beldie, A., Brain, C., Jakovljevic, M., Jarema, M., Karamustafalioglu, O., ... Thornicroft, G. (2015). Experience of stigma and discrimination reported by people experiencing the first episode of schizophrenia and those with a first episode of depression: The FEDORA project. International Journal of Social Psychiatry, 61(5), 438-445.

https://doi.org/10.1177/0020764014551941

Farrelly, S., Clement, S., Gabbidon, J., Jeffery, D., Dockery, L., Lassman, F., ... Thornicroft, G. (2014). Anticipated and experienced discrimination amongst people with schizophrenia, bipolar disorder and major depressive disorder: a cross sectional study. BMC Psychiatry, 14(1), 157. https://doi.org/10.1186/1471-244x-14-157

Ikwuka, U., Galbraith, N., Manktelow, K., Chen-Wilson, J., Oyebode, F., \& Muomah, R. C. (2016). Attitude towards mental illness in southeastern nigeria: The contradictions of a communitarian culture. Journal of Community Psychology, 44(2), 182-198. https://doi.org/10.1002/jcop.21749

Larkings, J. S., \& Brown, P. M. (2018). Do biogenetic causal beliefs reduce mental illness stigma in people with mental illness and in mental health professionals? A systematic review. International Journal of Mental Health Nursing, Vol. 27, pp. 928941. https://doi.org/10.1111/inm.12390

Lien, Y. J., \& Kao, Y. C. (2019). Public beliefs and attitudes toward schizophrenia and depression in Taiwan: A nationwide survey. Psychiatry Research, 273(January), 435-442. https://doi.org/10.1016/j.psychres.2019.01.062

Maulik, P. K., Devarapalli, S., Kallakuri, S., Tripathi, A.
P., Koschorke, M., \& Thornicroft, G. (2019). Longitudinal assessment of an anti-stigma campaign related to common mental disorders in rural India. British Journal of Psychiatry, 214(2), 90-95. https://doi.org/10.1192/bjp.2018.190

Ndetei, D. M., Mutiso, V., Maraj, A., Anderson, K. K., Musyimi, C., \& McKenzie, K. (2016). Stigmatizing attitudes toward mental illness among primary school children in Kenya. Social Psychiatry and Psychiatric Epidemiology, 51(1), 73-80. https://doi.org/10.1007/s00127-015-1090-6

Page, M. J., McKenzie, J. E., \& Higgins, J. P. T. (2018). Tools for assessing risk of reporting biases in studies and syntheses of studies: A systematic review. BMJ Open, Vol. 8, pp. 1-16. https://doi.org/10.1136/bmjopen-2017-019703

Reavley, N. J., Morgan, A. J., \& Jorm, A. F. (2017). Predictors of experiences of discrimination and positive treatment in people with mental health problems: findings from an Australian national survey. Social Psychiatry and Psychiatric Epidemiology, 52(3), 269-277. https://doi.org/10.1007/s00127-016-1301-9

Schomerus, G., Angermeyer, M. C., Baumeister, S. E., Stolzenburg, S., Link, B. G., \& Phelan, J. C. (2016). An online intervention using information on the mental health-mental illness continuum to reduce stigma. European Psychiatry, 32, 21-27. https://doi.org/10.1016/j.eurpsy.2015.11.006

Subica, A. M., Aitaoto, N., Sullivan, J. G., Henwood, B. F., Yamada, A. M., \& Link, B. G. (2019). Mental illness stigma among Pacific Islanders. Psychiatry Research, 273(January), 578-585. https://doi.org/10.1016/j.psychres.2019.01.077

Subramaniam, M., Abdin, E., Picco, L., Pang, S., Shafie, S., Vaingankar, J. A., ... Chong, S. A. (2017). Stigma towards people with mental disorders and its components - A perspective from multi-ethnic Singapore. Epidemiology and Psychiatric Sciences, Vol. 26, pp. 371-382. https://doi.org/10.1017/S2045796016000159

Svensson, B., \& Hansson, L. (2016). How mental health literacy and experience of mental illness relate to stigmatizing attitudes and social distance towards people with depression or psychosis: A cross-sectional study. Nordic Journal of Psychiatry, $70(4)$,

309-313. https://doi.org/10.3109/08039488.2015.11091 40

Townley, G., Brusilovskiy, E., \& Salzer, M. S. (2017). Urban and non-urban differences in community living and participation among individuals with serious mental illnesses. Social Science and Medicine, 177, 223-230. https://doi.org/10.1016/j.socscimed.2017.01.05 8

Xu, Z., Müller, M., Heekeren, K., Theodoridou, A., Dvorsky, D., Metzler, S., ... Rüsch, N. (2016). Selflabelling and stigma as predictors of attitudes towards help-seeking among people at risk of psychosis: 1-year follow-up. European Archives of Psychiatry and Clinical Neuroscience, 266(1), 79- 
R. P. ASTUTI ET AL.

82. https://doi.org/10.1007/s00406-015-0576-2 Zhuang, X. Y., Wong, D. F. K., Cheng, C. W., \& Pan, S. M. (2017). Mental health literacy, stigma and perception of causation of mental illness among Chinese people in Taiwan. International Journal of
Social Psychiatry, 63(6), 498-507. https://doi.org/10.1177/0020764017719303 